Wien klin Mag 2018 $\cdot 21: 240-245$ https://doi.org/10.1007/s00740-018-0250-1 Online publiziert: 28. September 2018 (c) Der/die Autor(en) 2018

CrossMark

\title{
Vanessa Stadlbauer-Köllner
}

Universitätsklinik für Innere Medizin, Abteilung für Gastroenterologie und Hepatologie, Medizinische Universität Graz, Graz, Österreich

\section{Probiotika bei Lebererkrankungen}

\section{Wirkmechanismen und klinische Effekte}

für Nahrungszwecke verwendet werden, durchlaufen einen Qualified-Presumption-of-Safety(QPS)-Prozess. Dabei wird festgestellt, ob die taxonomische Einheit bekannt ist, und bewertet, ob die Gesamtheit des Wissens über Pathogenität, Einsatzziele und Sicherheit ausreicht. Wenn die Voraussetzungen erfüllt sind, bekommt der Stamm QPS-Status. Listen mit den Keimen, die QPS Status haben, werden von der EFSA veröffentlicht. Um die Sicherheit zu gewährleisten, müssen die Produktionsprozesse kontaminationsfrei ablaufen, die Stämme müssen gegenüber gängiger Antibiotika sensibel und mittels gängigen diagnostischen Verfahren in Patientenproben nachweisbar sein. Außerdem sollten die Stämme genetisch so stabil sein, damit sie keine Pathogenitätsfaktoren oder Resistenzgene anderer Keime übernehmen.

Probiotika gelten generell als sicher, viele Stämme haben auch eine lange Tradition in der Nahrungsmittelindustrie. Allerdings gibt es immer wieder Berichte von Infektionen bis hin zur Sepsis durch probiotische Keime. Bei Patienten, die eine medikamentöse Immunsuppression benötigen oder die durch eine Grunderkrankung immunkompromittiert sind, stellt sich daher besonders die Frage nach der Sicherheit bei der Anwendung von Probiotika. In einer Metaanalyse von Studien, die Probiotika bei immunsupprimierten Erwachsenen (Infektion mit humanem Immundefizienzvirus [HIV], Organtransplantation, maligne Grunderkrankung, kritisch Kranke, Organerkrankungen mit immunsuppressivem Effekt, rheumatoide Arthritis, chronisch entzündliche Darmerkrankungen) eingesetzt hatten, wurde die Häufigkeit von Nebenwirkungen untersucht. In dieser Metaanalyse konnte kein Hinweis auf ein erhöhtes Risiko für Nebenwirkungen durch die Probiotika-Gabe gefunden werden, allerdings wird in vielen Studien unzureichend über Nebenwirkungen berichtet. Auch bei Leberzirrhose als häufigste erworbene Immunschwächeerkrankung ist der Einsatz von Probiotika als sicher zu beurteilen.

\section{Probiotika: Wirkung und Wirksamkeit}

Probiotika werden in Europa meistens als Nahrungsergänzungsmittel, diätetische Lebensmittel oder Nahrungsmittel vertrieben. Einzelne Stämme sind auch als Arzneimittel zugelassen; die $\mathrm{Zu}$ lassung erfolgte bereits vor mehreren Jahrzehnten. Ein klinischer Wirkungsnachweis nach den heutigen Kriterien der Arzneimittelzulassung ist bei Nahrungsergänzungsmitteln, diätetischen Lebensmitteln oder Nahrungsmitteln nicht notwendig bzw. auch von den regulierenden Behörden nicht gewünscht. Bislang wurde noch keinem Probiotikum ein „health claim“ zuerkannt. Dieses regulatorische Dilemma darf aber nicht dahingehend ausgelegt werden, dass Probiotika keine Wirkung haben. Im Gegenteil - zahlreiche Wirkungsweisen von probiotischen Keimen wurden bereits entschlüsselt. - Abb. 1 stellt die aktuell bekannten Wirkungsweisen von Probiotika dar, die bei Lebererkrankungen von Relevanz sein können. Ein probiotischer Stamm kann eine oder mehrere dieser Wirkungen haben. Die Kombination von mehreren Probiotika 


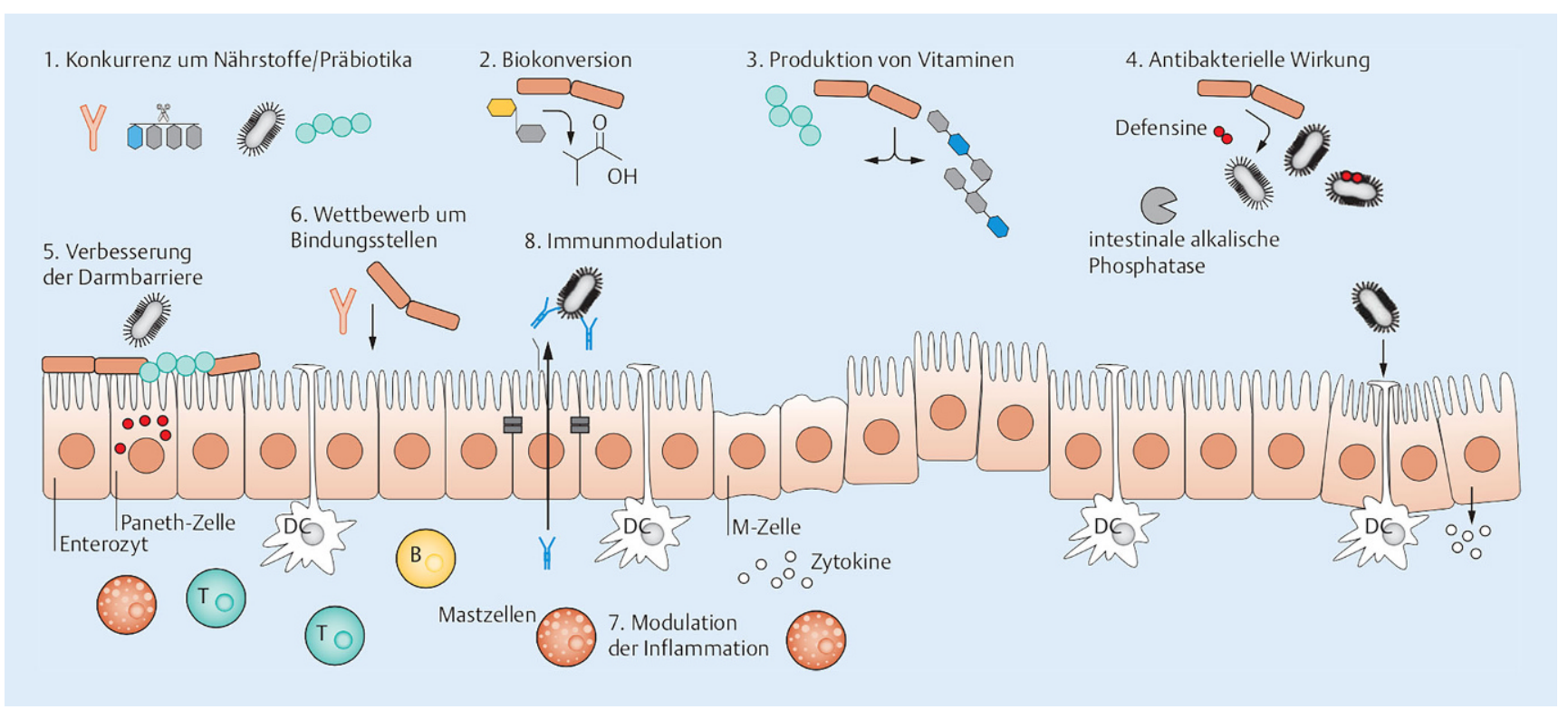

Abb. 1 \ Mögliche Wirkungsweisen von Probiotika (๑ Georg Thieme Verlag KG/with permission of the publisher)

- als Multi-Stamm oder Multi-SpeziesPräparate - kann durch additive und idealerweise synergistische Effekte die Wirksamkeit erhöhen.

\section{Anwendungsgebiete für Probiotika in der Hepatologie}

\section{Metabolische Lebererkrankungen}

Die nichtalkoholische Fettleber(NAFLD) ist eine stetig zunehmende Erkrankung, die in den USA bereits $25 \%$ der Bevölkerung betrifft und häufig mit dem metabolischen Syndrom assoziiert ist. Problematisch ist die Entwicklung einer nichtalkoholischen Steatohepatitis (NASH), die in weiterer Folge zur Entwicklung einer Leberzirrhose führt und das Risiko für die Entwicklung eines hepatozellulären Karzinoms erhöht. Dem Darm-Mikrobiom und der Darmbarriere kommt dabei eine Schlüsselrolle zu. Eine fett- und zuckerreiche Diät führt zu Veränderungen des Mikrobioms sowie vermehrter endogener Alkoholproduktion, stört die Darmbarriere und führt damit zu bakterieller Translokation (Übertritt von bakteriellen Bestandteilen oder Produkten in die portale Zirkulation). Die vermehrte bakterielle Translokation gemeinsam mit vermehrt einströmenden kurzkettigen Fettsäuren und anderen toxischen
Metaboliten aus dem Darm führt in der Leber zu Lipogenese und einer inflammatorischen Reaktion. Darüber hinaus bewirken die Mikrobiomveränderungen bei NAFLD eine Verminderung der Expression von Bestandteilen des Inflammasoms (zytosolischer Proteinkomplex in Makrophagen und neutrophilen Granulozyten), wodurch dann auch wieder vermehrt bakterielle Bestandteile in die Zirkulation gelangen und über Toll-likeRezeptor-Aktivierung eine inflammatorische Reaktion hervorrufen. Auch für Glukose wurde kürzlich gezeigt, dass eine Hyperglykämie über den GLUT2Rezeptor auf Enterozyten die Darmpermeabilität erhöhen und ebenso eine bakterielle Translokation bewirken.

Aus diesen Erkenntnissen entstand die Hypothese, dass Probiotika einen positiven Einfluss auf die Steatose oder Steatohepatitis beim Menschen haben könnten. $\mathrm{Zu}$ dieser Fragestellung wurden mehrere randomisiert-kontrollierte Studien durchgeführt, allerdings variieren die Studienprotokolle stark bezüglich Patientenkohorte, verwendetem Produkt, Dosis und Dauer und sind daher schwer vergleichbar. Mehrere Metaanalysen (- Tab. 1) zeigen einen positiven Einfluss auf Surrogatparameter wie Leberwerte oder Biomarker der Inflammation. Die meisten Studien untersuchten bisher leider keine harten
Endpunkte, wie z. B. die Leberhistologie. Manche Studienergebnisse sind auch widersprüchlich - es kam mit bestimmten Produkten zu Gewichtszunahmen bzw. vermehrter Fetteinlagerung in die Leber. In Kombination mit anderen Therapien zeigte sich bisher eine synergistische Wirkung zusammen mit Metformin, allerdings kein zusätzlicher Effekt durch Probiotika bei bariatrischer Chirurgie, very-low-calorie Diäten oder deutlicher Fruktosereduktion. Zusammenfassend scheinen die bislang untersuchten Probiotika bei NAFLD und NASH messbare, aber eher geringe Effekte zu haben. Um eine allgemeingültige Empfehlung zur Verwendung von Probiotika in der Therapie der nichtalkoholischen Steatose bzw. Steatohepatitis zu rechtfertigen, ist es daher noch zu früh. Die Entwicklung von auf die Pathogenese abgestimmten Next-generation-Probiotika, adäquat designte große Studien, die Kombinationen mit anderen effektiven Therapien und die Untersuchung harter Endpunkte muss dabei das Ziel sein.

\section{Leberzirrhose}

Das Darm-Mikrobiom ist bei Leberzirrhose massiv in seiner Zusammensetzung gestört. Typisch sind eine Verminderung der Diversität und ein Überwiegen von pathogenen Keimen. Auffällig 
ist insbesondere eine Oralisierung des Darm-Mikrobioms - es finden sich Mundkeime wie Veillonella und Streptococcus in vermehrter Anzahl im Darm, die durch Medikamente, wie z.B. Protonenpumpenhemmer, noch verstärkt wird. Parallel dazu nimmt die Anzahl an Bakterien, denen positive Wirkungen auf den Menschen zugeschreiben werden, wie beispielsweise Faecalibacterium prausnizii ab. Die Ursachen dafür sind vielfältig: Alkohol und sein Abbauprodukt Azetaldehyd schädigen das Mikrobiom und die Darmbarriere; die $\mathrm{Zu}$ sammensetzung der Nahrung (s. oben) beeinflusst Mikrobiom und Darmbarriere; aber auch der portale Hypertonus und Medikamente spielen eine Rolle. Daher erscheint auch hier das Konzept der Modulation des Darm-Mikrobioms und/oder der Darmpermeabilität attraktiv. Probiotika wurden vor allem zur Therapie von Komplikationen der Leberzirrhose (hepatische Enzephalopathie, Infektionen) untersucht. Rezent gibt es auch Hinweise, dass Probiotika die Leberfunktion verbessern können.

Schon seit mehr als 40 Jahren werden Probiotika als therapeutische Strategie bei hepatischer Enzephalopathie untersucht. In kleinen, nichtkontrollierten Studien konnte bei bis zu drei Viertel der Patienten eine klinische Verbesserung nachgewiesen werden. Mehrere größere, randomisierte, kontrollierte Studien zeigten ebenfalls einen positiven Effekt von Probiotika, Präbiotika oder der Kombination aus beiden auf den Schweregrad der hepatischen Enzephalopathie, psychometrische Tests und Laborwerte. Ebenso konnte das Risiko für das Auftreten einer neuen Episode einer hepatischen Enzephalopathie reduziert werden. Aus diesen Ergebnissen lässt sich ableiten, dass Probiotika wahrscheinlich in der Prophylaxe und Behandlung der hepatischen Enzephalopathie wirksam sind, allerdings ist aufgrund der unterschiedlichen Studiendesigns (manche verglichen mit Placebo, manche mit Laktulose) und unterschiedlicher verwendeter Produkte (manche sind auch nicht kommerziell erhältlich) sowie der fraglichen Überlegenheit gegenüber der Standardtherapie die therapeutische Relevanz dieser Therapieform noch nicht

Wien klin Mag 2018 · 21:240-245 https://doi.org/10.1007/s00740-018-0250-1

(c) Der/die Autor(en) 2018

\section{Stadlbauer-Köllner \\ Probiotika bei Lebererkrankungen. Wirkmechanismen und klinische Effekte}

\section{Zusammenfassung}

Die Mikrobiomforschung gewinnt in den letzten Jahren zunehmend an Bedeutung. Insbesondere bei Lebererkrankungen werden Veränderungen des Mikrobioms häufig beobachtet und als pathophysiologisch relevant angesehen. Strategien zur Modulation des Mikrobioms sind daher ein vielversprechender neuer therapeutischer Ansatz. Probiotika sind eine Möglichkeit, das Mikrobiom zu beeinflussen. In dieser Übersichtsarbeit wird die Sicherheit und Wirksamkeit von Probiotika allgemein diskutiert und dann im Speziellen auf die vorhandenen klinischen Daten bei Fettlebererkrankung sowie bei
Leberzirrhose eingegangen. Probiotika können insbesondere bei der Behandlung von Komplikationen der Leberzirrhose wirksam sein, es gibt allerdings noch Bedarf an großen, qualitativ hochwertigen Studien um das therapeutische Potenzial und die möglichen Nebenwirkungen von Probiotika zu untersuchen.

Schlüsselwörter

Mikrobiom - Therapie - Sicherheit . Leberzirrhose · Nicht-alkoholische Fettlebererkrankung

\section{Probiotics For Therapy of Liver Diseases. Mechanisms of Action and Clinical Effects}

\section{Abstract}

Microbiome research has gained considerably in importance in recent years. Particularly in liver diseases, microbiome changes have frequently been observed and are considered as pathophysiologically relevant. Therefore, strategies to modulate the microbiome represent a promising new therapeutic concept. Probiotics are one possibility to modulate the microbiome. In this review, safety and efficacy of probiotics will be discussed in general and clinical data on the effect of probiotics on non-alcoholic fatty liver disease and liver cirrhosis will be discussed in greater detail. Probiotics have been shown to be beneficial in the treatment of complications of liver cirrhosis; however, large, high-quality studies are needed to study the therapeutic potential and possible side effects of this therapy.

\section{Keywords}

Microbiome - Therapy · Safety · Liver cirrhosis . Non-alcoholic liver disease klar definiert. Daher gibt es aktuell auch noch keine Empfehlung einer Fachgesellschaft zur Verwendung von Probiotika, sondern es müssen weitere, qualitativ hochwertige Studien durchgeführt werden. Auch ein Cochrane Review von Februar 2017 kommt zu dem Ergebnis, dass Probiotika zwar wahrscheinlich wirksam sind, aber noch mehr Daten notwendig sind (• Tab. 1).

Ein weiteres großes klinisches Problem ist die Tatsache, dass Patienten mit Leberzirrhose ein erhöhtes Infektionsrisiko haben. Die Leberzirrhose geht mit einer schwerwiegenden Störung des angeborenen Immunsystems einher und kann daher als die häufigste erworbene Immunschwächeerkrankung angesehen werden. Diese Funktionsstörung ist mit einer erhöhten Rate an Infektionen, vermehrtem Auftreten an Organversagen und erhöhter Mortalität vergesellschaftet. Die Hälfte aller ungeplanten stationären Aufnahmen von Leberzirrhose-Patienten erfolgt aufgrund von Infektionen, weitere 30-35\% entwickeln während eines stationären Aufenthalts eine Infektion. Infektionen sind auch ein häufiger Trigger für die Dekompensation und die Entwicklung eines akut-auf-chronischen Leberversagens. Als Ursachen der Immundysfunktion werden eine Überstimulation mit bakteriellen Produkten (z. B. Endotoxine), dysfunktionales Albumin und andere Serumproteine, genetische Ursachen 


\begin{tabular}{|c|c|c|c|}
\hline Referenz & Indikation & Anzahl Studien/Patienten & Ergebnis \\
\hline [1] & NAFLD & $7 / 276$ & Verbesserung von BMI, AST, ALT, HOMA-IT, Steatose (Ultraschall) \\
\hline [2] & NAFLD & $9 / 535$ & Verbesserung von HOMA-IR, Gesamt-Cholesterin, LDL \\
\hline [3] & NAFLD/NASH & $4 / 134$ & Verbesserung von AST, ALT, TNF-a, Gesamt-Cholesterin, HOMA-IR \\
\hline \multirow[t]{2}{*}{ [4] } & \multirow[t]{2}{*}{$\begin{array}{l}\text { Hepatische } \\
\text { Enzephalopathie }\end{array}$} & \multirow[t]{2}{*}{$21 / 1420$} & $\begin{array}{l}\text { Probiotika möglicherweise effektiv zur Prophylaxe und Therapie der hepati- } \\
\text { schen Enzephalopathie, Verbesserung der Lebensqualität und Senkung der } \\
\text { Serum-Ammoniakspiegel }\end{array}$ \\
\hline & & & Kein Einfluss auf Mortalität \\
\hline \multirow[t]{2}{*}{ [5] } & \multirow[t]{2}{*}{$\begin{array}{l}\text { Hepatische } \\
\text { Enzephalopathie }\end{array}$} & \multirow[t]{2}{*}{$14 / 1152$} & $\begin{array}{l}\text { Probiotika besser als Placebo bei der Reduktion der Hospitalisierungsrate, } \\
\text { Verbesserung der minimalen hepatischen Enzephalopathie und Prävention der } \\
\text { Progression }\end{array}$ \\
\hline & & & Kein Einfluss auf Mortalität \\
\hline [6] & $\begin{array}{l}\text { Hepatische Enze- } \\
\text { phalopathie }\end{array}$ & $6 / 496$ & $\begin{array}{l}\text { Probiotika reduzieren das Risiko, eine overte hepatische Enzephalopathie zu } \\
\text { entwickeln }\end{array}$ \\
\hline [7] & $\begin{array}{l}\text { Minimale hepati- } \\
\text { sche Enzephalopa- } \\
\text { thie }\end{array}$ & $14 / 1132$ & $\begin{array}{l}\text { Probiotika vermindern Serum-Ammonika- und Endotoxinspiegel, verbessern } \\
\text { die minimale hepatische Enzephalopathie und verhindern die Progression zu } \\
\text { einer overten hepatischen Enzephalopathie }\end{array}$ \\
\hline [8] & $\begin{array}{l}\text { Verbesserung von } \\
\text { Leberwerten }\end{array}$ & $17 / 497$ & Verbesserung von AST, ALT, GGT \\
\hline [9] & $\begin{array}{l}\text { Infektionen nach Le- } \\
\text { bertransplantation }\end{array}$ & $4 / 246$ & $\begin{array}{l}\text { Prä- und Probiotika vor bzw. bei der Lebertransplantation senken das postope- } \\
\text { rative Infektionsrisiko }\end{array}$ \\
\hline \multicolumn{4}{|c|}{$\begin{array}{l}\text { ALT Alanin-Aminotransferase; AST Aspartat-Aminotransferase; BMI Body Mass Index; GGT gamma-Glutamyltransferse; HOMA_IR Homeostatic Model } \\
\text { Assessment for Insulin Resistance; LDL low density lipoprotein; NAFLD nichtalkoholische Fettlebererkrankung; NASH nicht-alkoholische Steatohepatitis; } \\
\text { TNF-alpha Tumornekrosefaktor alpha }\end{array}$} \\
\hline
\end{tabular}

und möglicherweise auch Gallensäuren diskutiert. Die Immundysfunktion ist nach einer Lebertransplantationen besonders ausgeprägt, da zusätzlich zur bestehenden Leberzirrhose noch die große Operation ebenfalls zu einer erhöhten Darmpermeabilität und vermehrter bakterieller Translokation führt, die auch nach einer Transplantation noch länger nachweisbar ist. Eine Metaanalyse von 4 kontrollierten Studien (•Tab. 1) kam zu dem Ergebnis, dass eine perioperative Gabe von Probiotika das Infektionsrisiko nach Lebertransplantation senken kann. Auch dieser Ansatz wurde noch nicht in multizentrischen Studiensettings untersucht, und die probiotische Infektionsprophylaxe bei Lebertransplantation findet sich daher noch nicht in Empfehlungen von Fachgesellschaften.

Je weiter fortgeschritten eine Lebererkrankung ist, desto wahrscheinlicher sind Komplikationen und desto höher ist die Mortalität. Eine Verbesserung der Leberfunktion bei fortgeschrittener Leberzirrhose ist nur schwierig zu erreichen. Die Behandlung der Grunderkrankung kann nicht immer in zufriedenstellendem Maß erreicht werden, und eine Le- bertransplantation, die aufgrund von $\mathrm{Pa}$ tientenfaktoren und Mangel an Spenderorganen auch nicht für jeden Patienten in Frage kommt, sind die heute verfügbaren Möglichkeiten um die Leberfunktion per se zu verbessern. Unterschiedliche Probiotika zeigten in Pilotstudien eine Besserung von Leberwerten, Endotoxinspiegel und des Child-Pugh-Scores als Summenscore der Leberfunktion. Eine rezente Metaanalyse zeigte, dass insbesondere Synbiotika (Kombination aus Pro- und Präbiotika), wenn sie für mehr als 8 Wochen verwendet werden, die Leberwerte (Aspartat-Amoniotransferase [AST], Alanin-Aminotransferase [ALT], Gamma-Glutamyltransferase [GGT]) verbessern können. Nachdem diese Metaanalyse auch Studien an Lebergesunden und PatientInnen mit chronischen Lebererkrankungen ohne Zirrhose einschließt, lässt sie keine Schlussfolgerung über den Effekt von Probiotika auf die Leberfunktion bei Leberzirrhose zu. Eine randomisierte, placebokontrollierte Studie unserer Arbeitsgruppe konnte zeigen, dass ein Multispeziesprobiotikum die Leberfunktion, die angeborene Immunabwehr und die Lebensqualität verbessert.

\section{Cholestatische Lebererkrankungen}

Auch bei cholestatischen Lebererkrankungen nimmt die Evidenz bezüglich der Rolle des Mikrobioms und der DarmLeber-Achse zu. Gallensäuren spielen in der Pathogenese von cholestatischen Lebererkrankungen eine wichtige Rolle und stehen mit dem Mikrobiom in enger Wechselbeziehung: Das Mikrobiom determiniert die Gallensäurezusammensetzung (Bildung der sekundären Gallensäuren) und die Gesamtgröße des Gallensäurepools. Gallensäuren steuern die Mikrobiom-Zusammensetzung, da sie einerseits antibakterielle Eigenschaften haben, während andererseits bestimmte Keime darauf adaptiert sind, durch Gallensäuren besser zu wachsen (z. B. verschiedene Clostridium- und Ruminococcus-Spezies). Bei primär sklerosierender Cholangitis findet man interessanterweise eine höhere Diversität des Mikrobioms und eine Verschiebung in der Zusammensetzung des Mikrobioms. Therapien die auf eine Modulation des Darm-Mikrobioms abzielen, sind daher attraktiv. Bezüglich Probiotika gibt es bisher nur eine Studie, die keinen Effekt des verwendeten Produktes auf die 
Cholestaseparameter zeigte. Auch bei der primär biliären Cholangitis zeigen auf Speziesebene des Mikrobioms einige Verschiebungen, wie z.B. eine Abnahme von günstigen Keimen und eine Zunahme von potenziell pathogenen Keimen im Sinne einer Oralisierung. Die Änderungen in der MikrobiomZusammensetzung sind mit funktionellen Veränderungen assoziiert. Es ist noch unklar, ob die Mikrobiom-Veränderungen die Erkrankung triggern oder Ausdruck der Erkrankung sind. Ebenso fehlen therapeutische Ansätze in dieser Indikation noch.

\section{Hepatozelluläres Karzinom}

Das hepatozelluläre Karzinom (HCC) tritt zu über $90 \%$ in einer zirrhotischen Leber auf. Störungen der Darm-LeberAchse werden zunehmend als Pathomechanismus diskutiert. Aus dem Darm kommen zahlreiche bakterielle Bestandteile in die Leber, die dort Toll-likeRezeptoren aktivieren. Deren Signalwege sind in die Tumorprogression involviert. In einer Pilotstudie konnte RNA und DNA von verschiedenen Bakterien im Lebergewebe nachgewiesen werden, dies könnte auf eine direkte Rolle des Mikrobioms in der Tumorentstehung hindeuten. Tierexperimentell konnte auch schon ein direkter Zusammenhang zwischen bakteriellen Produkten (z.B. Endotoxin) und Tumorwachstum nachgewiesen werden. Außerdem scheint die Wirksamkeit von modernen Antitumortherapien, wie z. B. Checkpoint-Inhibitoren, eng mit der Struktur und Funktion des Darmmikrobioms zusammenzuhängen. In Tierexperimenten konnten ein positiver Einfluss von Probiotika im Sinne einer Verringerung des Tumorwachstumes und eine Verbesserung von therapieassoziierten Nebenwirkungen gezeigt werden. Studien am Menschen fehlen aber noch.

\section{Fazit für die Praxis}

Die Bedeutung der Darm-Leber-Achse und damit des Darm-Mikrobioms in der Pathogenese von Lebererkrankungen und deren Komplikationen ist unumstritten. Durch die technischen Fort- schritte in der Analyse des Mikrobioms können heute funktionelle Zusammenhänge besser entschlüsselt werden. Probiotika sind eine vielversprechende und sichere Strategie zur Beeinflussung der Darm-Leber-Achse. Die besten Daten gibt es zur Therapie von Komplikationen, wie der hepatischen Enzephalopathie oder Infektionen. Eine Verbesserung der Leberfunktion ist ein vielversprechendes Therapieziel, das noch genauer untersucht werden muss. Es besteht noch Bedarf an großen, qualitativ hochwertigen Studien, um das therapeutische Potenzial und die möglichen Nebenwirkungen von Probiotika zu untersuchen.

\section{Korrespondenzadresse}

Assoz. Prof. Dr. med. univ. Vanessa Stadlbauer-Köllner

Universitätsklinikfür Innere Medizin, Abteilung für Gastroenterologie und Hepatologie, Medizinische Universität Graz

Auenbruggerplatz 15, 8036 Graz, Österreich vanessa.stadlbauer@medunigraz.at

Funding. Open access funding provided by Medical University of Graz.

\section{Einhaltung ethischer Richtlinien}

Interessenkonflikt. V. Stadlbauer-Köllner erhielt Forschungsförderungen, Vortragshonorare und/oder Reisekostenersatz von den Firmen Fresenius Austria, Winclove GmbH und Institut Allergosan.

Dieser Beitrag beinhaltet keine vom Autor durchgeführten Studien an Menschen oder Tieren.

Open Access. Dieser Artikel wird unter der Creative Commons Namensnennung 4.0 International Lizenz (http://creativecommons.org/licenses/by/4.0/deed. de) veröffentlicht, welche die Nutzung, Vervielfältigung, Bearbeitung, Verbreitung und Wiedergabe in jeglichem Medium und Format erlaubt, sofern Sie den/die ursprünglichen Autor(en) und die Quelle ordnungsgemäßnennen, einen Link zur Creative Commons Lizenz beifügen und angeben, ob Änderungen vorgenommen wurden.

\section{Literatur}

1. Laveka AS et al (2017) Euroasian J Hepatogastroenterol 7(2):130-137. https://doi.org/10.5005/jpjournals-10018-1233

2. Gao X et al (2016) Hepatol Res 46(12):1226-1233. https://doi.org/10.1111/hepr.12671

3. Ma YY et al (2013) World J Gastroenterol 19(40):6911-6918. https://doi.org/10.3748/wjg. v19.i40.6911
4. Dalal R et al (2017) Cochrane Database Syst Rev. https://doi.org/10.1002/14651858.CD008716. pub3

5. Saab S et al (2016) Liver Int 36(7):986-993. https:// doi.org/10.1111/liv.13005

6. Xu J et al (2014) Hepatobiliary Pancreat Dis Int 13(4):354-360

7. Cao Q et al (2018) Hepatobiliary Pancreat Dis Int 17(1):9-16

8. Khalesi S (2017) Eur J Nutr. https://doi.org/10. 1007/s00394-017-1568-y

9. Sawas T (2015) Clin Gastroenterol Hepatol. 2015 Sep;13(9):1567-74.e3. https://doi.org/10.1016/j. cgh.2015.05.027 
Hier steht eine Anzeige.

\section{曾 Springer}

